\title{
EU-Weißbuch Erneuerbare Energien und die Ergebnisse von Kyoto
}

\author{
W. Hein ${ }^{1}$
}

\begin{abstract}
Das Kyoto-Protokoll zur Internationalen Klimakonvention sieht eine Reduktion der Treibhausgase (vor allem Kohlendioxid) in der EU und den meisten assoziierten Staaten um $8 \%$ von 1990 bis 2010 vor. Das Weißbuch Erneuerbare Energien der Europäischen Kommission sieht eine Verdoppelung des Anteils Erneuerbare Energie am Energieverbrauch der EU von jetzt $6 \%$ auf $12 \%$ bis 2010 vor. Diese beiden Ziele können gemeinsam angesteuert werden.
\end{abstract}

Schlüsselwörter: Erneuerbare Energie; Kyoto-Ziele; EU-Weißbuch Erneuerbare Energie; Energiestatistik

EU-Whitebook on renewable energies and the results of Kyoto. The Kyoto Protocol to the United Nations Framework Convention on Climate Change gives a target for the reduction of greenhouse gases in the EU and most associated states of $8 \%$ from 1990 levels to 2010. The European Commissions Whitebook on Renewable Energy is setting a target of doubling the share of Renewable Energy from $6 \%$ now to $12 \%$ in 2010 for the European Union. There is no contradiction between these targets.

Keywords: renewable energy; Kyoto-targets; EU-whitebook on renewable energy; energy statistics

\section{Die Ergebnisse von Kyoto}

Schon in den achtziger Jahren ist der Umweltschutz ein vorrangiges politisches Ziel der Europäischen Union geworden und muß bei Konflikten mit den Zielen der Liberalisierung des Binnenmarktes und der europäischen Agrarpolitik nicht mehr automatisch zurückstehen. Die EU hat sich zur treibenden Kraft bei der Konzeption und Verhandlung internationaler Umweltschutzabkommen entwickelt und dies sowohl bei der Erarbeitung der Abkommen zum Schutz der Ozon-Schicht (Ausstieg aus FCKW: Wiener Vertrag, Montreal-Protokoll) wie auch der Abkommen zur Begrenzung menschenverursachter Klimaveränderungen (Klimakonvention Rio 1992, Kyoto-Protokoll 1997) unter Beweis gestellt.

Die EU ist in die Verhandlungen bei der Klimakonferenz von Kyoto mit einem Ziel von $15 \%$ Reduktion der Treibhausgase bis 2010 bezogen auf 1990 gegangen, während die USA nur eine Stabilisierung in Aussicht stellten und Japan eine Reduktion um $5 \%$. Das unter höchsten politischen Anstrengungen des US-Präsidenten Clinton und der EU-Regierungschefs (angeführt von Tony Blair) sowie dem japanischen Minister-

\footnotetext{
' Min.-Rat Dipl.-Ing. Wolfgang Hein, Bundeskanzleramt, Hohenstaufengasse 3, A-1010 Wien.
}

präsidenten Hashimoto, errungene Kyoto-Protokoll sieht nun für die EU eine Reduktion um $8 \%$, für die USA um $7 \%$ und für Japan um $6 \%$ vor.

Das bei weitem bedeutendste Treibhausgas ist dabei Kohlendioxid, das größtenteils aus der Verbrennung von Kohle, Erdöl und Erdgas stammt. Damit ist auch der wichtigste Ansatzpunkt für die Erreichung des Kyoto-Ziels klar: die Energiepolitik. Ohne eine deutliche Reduktion des Verbrauchs fossiler Energien ist das Ziel nicht erreichbar.

Diese Reduktion muß von zwei Seiten angegangen werden: Steigerung der Energieeffizienz (weniger Leerlauf, bessere Wirkungsgrade, bessere Isolation) und Umstieg auf erneuerbare Energien (Biomasse, Wasser, Wind, Sonne, Erdwärme). Eine wirkliche und dauerhafte Verhinderung von weiteren menschenverursachten Klimaveränderungen ist letztlich nur über die vollständige Ablöse der fossilen Energien durch erneuerbare vorstellbar.

Dabei kann nicht gewartet werden, bis die erneuerbaren Energien unter den derzeitigen unfairen Rahmenbedingungen billiger sind als die fossilen: $\mathrm{zu}$ viele Möglichkeiten hat das Big Business, für sich günstige Bedingungen zu prolongieren und auf jede technische Weiterentwicklung bei den Erneuerbaren mit Preissenkungen bei den Fossilen zu reagieren. 
Entgegen dem derzeit vorherrschenden Trend, sich selbst $\mathrm{zu}$ entmachten und alle Verantwortung der Markwirtschaft zu überlassen, ist die Politik in einer solchen Situation gefordert.

\section{Das Weißbuch Erneuerbare Energieträger der Europäischen Kommission}

Eine gute Analyse der Situation im Energiebereich und eine Sammlung von Anregungen für die Politik ist das von der Europäischen Kommission vorgelegte Weißbuch Erneuerbare Energien für eine Gemeinschaftsstrategie samt einem Aktionsplan für die Europäischen Union und ihre Mitglieder.

Gemäß den Angaben des Europäischen Statistischen Amtes beträgt der Anteil erneuerbarer Energieträger am Bruttoinlandsenergieverbrauch 5,3\% für 1995, wobei das Vereinigte Königreich mit $0,7 \%$ und Belgien mit $1 \%$ die Schlußlichter und Schweden mit $25,4 \%$ und Österreich mit $24,3 \%$ die Anführer sind (Tab. 1).

Tabelle 1. Anteil erneuerbarer Energie am Bruttoinlandsverbrauch (nach EUROSTAT) in Prozent für 1990 und 1995

\begin{tabular}{lrr}
\hline & 1990 & 1995 \\
\hline Österreich & 22,1 & 24,3 \\
Belgien & 1,0 & 1,0 \\
Dänemark & 6,3 & 7,3 \\
Finnland & 18,9 & 21,3 \\
Frankreich & 6,4 & 7,1 \\
Deutschland & 1,7 & 1,8 \\
Griechenland & 7,1 & 7,3 \\
Großbritannien \& Nord-Irland & 0,5 & 0,7 \\
Irland & 1,6 & 2,0 \\
Italien & 5,3 & 5,5 \\
Luxemburg & 1,3 & 1,4 \\
Niederlande & 1,3 & 1,4 \\
Portugal & 17,6 & 15,7 \\
Spanien & 6,7 & 5,7 \\
Schweden & 24,7 & 25,4 \\
\hline Europäische Union & 5,0 & 5,3 \\
\hline
\end{tabular}

Doch schon diese Statistik bestätigt die Reserven des Apparats gegenüber den Erneuerbaren: nur die Biomasse paßt in bisherige Schemas, sie kann wie Kohle als fester Brennstoff behandelt werden, es gibt empirische Wirkungsgrade bei der Herstellung von Wärme und Strom. Allerdings wird viel Holz ohne Einschaltung des Handels von Forst- und Holzwirtschaft selbst genutzt und ist daher statistisch schwer erfaßbar.

Bei der Wasserkraft wird nach EUROSTAT die erzeugte Elektrizität als Bruttoenergie gerechnet, es gibt keinen Wirkungsgrad bei der Umwandlung von
Wasserkraft in elektrische Energie. Somit schlägt Strom aus Wasserkraft in der Bruttoenergiestatistik nur zu etwa einem Drittel zu Buche im Vergleich zu Strom aus Kernenergie, da es dort einen Wirkungsgrad bei der Umwandlung von Wärme in Strom gibt wie bei fossil befeuerten Kraftwerken. Dasselbe gilt für Strom aus Windkraft und Photovoltaik.

Würde Strom aus Wasserkraft nach der Substitutionsmethode wie Strom aus thermischen Kraftwerken bewertet, so läge der Anteil erneuerbarer Energie schon für 1995 in der EU bei 8,1\%.

Eine faire Vorgangsweise für Wasserkraft wäre eventuell, das theoretische Wasserkraftpotential eines bestimmten Flußabschnitts mit dem daraus erzeugten Strom in Relation zu setzen und daraus einen Wirkungsgrad zwischen Primärenergie Wasserkraft und Sekundärenergie Strom zu errechnen. Bei Windenergie und Photovoltaik könnte man ähnlich vorgehen.

Bei thermischer Solarenergienutzung ist derzeit auch kein Wirkungsgrad zwischen der Sonneneinstrahlung und dem Wärmegehalt des damit erzeugten warmen Wassers vorgesehen, letzterer wird gleich Bruttoenergie gesetzt. So schlagen die immerhin 6,5 Mio. $\mathrm{m}^{2}$ Sonnenkollektoren in der EU nur mit $0,02 \%$ des Bruttoenergieverbrauchs zu Buche.

Österreich hätte bei fairer Bewertung der Wasserkraft, der Sonnenkollektoren und Wärmepumpen und guter Schätzung der Biomassenutzung jetzt schon etwa $30 \%$ erneuerbaren Anteil am Primärenergieverbrauch.

Das Weißbuch gibt jedoch abseits dieser statistischen Schwierigkeiten einen guten Überblick über die Verbreitung der erneuerbaren Energienutzung und ihrer Potentiale. Demnach könnte die Stromerzeugung aus erneuerbaren Energieträgern in der EU von 337 TWh (entsprechend 14,3\%) in 1995 auf 675 TWh für 2010 angehoben werden.

Große Wasserkraft würde dabei von $270 \mathrm{TWh}$ auf 300 TWh Jahreserzeugung anwachsen, kleine Wasserkraft von $37 \mathrm{TWh}$ auf $55 \mathrm{TWh}$. Windkraft würde sich von $4 \mathrm{TWh}$ auf $80 \mathrm{TWh}$ entwickeln, Photovoltaik von $0,03 \mathrm{TWh}$ auf $3 \mathrm{TWh}$. Strom aus Erdwärme verdoppelt sich von 3,5 TWh auf 7 TWh pro Jahr, der aus Biomasse verzehnfacht sich von $22,5 \mathrm{TWh}$ auf 230 TWh.

Wärmeerzeugung aus erneuerbaren Energiequellen könnte sich von $38 \mathrm{Mio}$. $\mathrm{t}$ Rohöleinheiten aus Biomasse 1995 bis 2010 etwa verdoppeln, bei Erdwärme von 0,4 Mio. t RöE auf 1 Mio. $t$ RöE ebenso, thermische Sonnenkollektoren könnten statt 0,26 Mio. t RöE in 1995 etwa 4 Mio. $t$ RöE für 2010 liefern. Aus passiver Sonnenenergienutzung durch geeignete Architektur könnten weitere 35 Mio. t RöE hinzukommen (Tab. 2). 
Tabelle 2. Anteil erneuerbarer Energie am Gesamtverbrauch in der EU (in Mio. $t$ RöE)

\begin{tabular}{|c|c|c|c|c|c|c|c|c|}
\hline & \multicolumn{4}{|c|}{1995} & \multicolumn{4}{|c|}{2010} \\
\hline & EUROSTAT & $\begin{array}{l}\text { Anteil } \\
\text { in } \%\end{array}$ & $\begin{array}{l}\text { Substitutions- } \\
\text { prinzip }\end{array}$ & $\begin{array}{l}\text { Anteil } \\
\text { in } \%\end{array}$ & EUROSTAT & $\begin{array}{l}\text { Anteil } \\
\text { in } \%\end{array}$ & $\begin{array}{l}\text { Substitutions- } \\
\text { prinzip }\end{array}$ & $\begin{array}{l}\text { Anteil } \\
\text { in } \%\end{array}$ \\
\hline Bruttoenergie & 1366 & 100 & 1409 & 100 & 1583 & 100 & 1633 & 100 \\
\hline $\begin{array}{l}\text { Wasser } \\
\text { Wind } \\
\text { PV } \\
\text { Biomasse } \\
\text { Erdwärme } \\
\text { Solar-WW }\end{array}$ & $\begin{array}{c}26,4 \\
0,35 \\
0,002 \\
44,8 \\
2,5 \\
0,26\end{array}$ & $\begin{array}{l}1,9 \\
0,02 \\
0 \\
3,3 \\
0,2 \\
0,02\end{array}$ & $\begin{array}{c}67,5 \\
0,9 \\
0,006 \\
44,8 \\
1,2 \\
0,26\end{array}$ & $\begin{array}{l}4,8 \\
0,06 \\
0 \\
3,1 \\
0,1 \\
0,02\end{array}$ & $\begin{array}{c}30,6 \\
6,9 \\
0,26 \\
135 \\
5,2 \\
4\end{array}$ & $\begin{array}{l}1,9 \\
0,44 \\
0,02 \\
8,5 \\
0,33 \\
0,25\end{array}$ & $\begin{array}{c}78,1 \\
17,6 \\
0,7 \\
135 \\
2,5 \\
4\end{array}$ & $\begin{array}{l}4,8 \\
1,07 \\
0,05 \\
8,3 \\
0,15 \\
0,24\end{array}$ \\
\hline $\begin{array}{l}\text { Erneuerb. } \\
\text { Energie }\end{array}$ & 74,3 & 5,44 & 14,7 & 8,1 & 182 & 11,5 & 238,1 & 14,6 \\
\hline Passiv solar & & & & & 35 & 2,2 & 35 & 2,1 \\
\hline
\end{tabular}

Das Weißbuch argumentiert ausführlich die Vorteile und Chancen der Nutzung Erneuerbarer Energiequellen für Umwelt und Beschäftigung und nennt die wesentlichen politischen Strategien zur Marktdurchdringung. Dies sind besserer Zugang zum Elektrizitätsmarkt, Steuer- und Finanzmaßnahmen, Initiativen für Bioenergie im Verkehrsbereich sowie zur Wärme- und Stromerzeugung und schließlich Vorschriften im Gebäudebereich.

Das Weißbuch nennt die wesentlichen Ziele und Instrumente der Europäischen Gemeinschaft und wie sie mit einer forcierten Politik zugunsten erneuerbarer Energie in Einklang gebracht werden können.

Im Aktionsplan wird vorgeschlagen, bis zum Jahr 2010 eine Kampagne für den Durchbruch erneuerberer Energien zu machen, die beispielhaft die Errichtung von einer Million Photovoltaikanlagen durch die EU und ihre Mitglieder vorsieht, davon die Hälfte in der EU und die Hälfte in Entwicklungsländern, insgesamt 3000 MW. Nicht viel im Vergleich mit der heute vorhandenen gesamten Stromerzeugungskapazität, sehr ambitioniert aber, wenn man es mit der Welterzeugung von Photovoltaik-Modulen von $125 \mathrm{MW}$ in 1997 in Relation setzt.

Weiters wird vorgeschlagen, bis 2010 große Windenergieparks mit insgesamt $10000 \mathrm{MW}$ in der EU zu errichten, was nicht so ambitioniert erscheint in Rela- tion zum schon vorhandenen: Deutschland hat schon etwa $2000 \mathrm{MW}$ und Dänemark etwa $1000 \mathrm{MW}$ Windenergiekapazität.

Der dritte Punkt dieser Kampagne sieht 10000 MWBiomasse-Anlagen vor, der vierte die beispielhafte Versorgung von 100 Gemeinden in der EU mit erneuerbaren Energien.

Mit Hilfe dieser und vieler anderer Maßnahmen im Rechtsbereich und im Förderungsbereich soll bis 2010 eine Verdoppelung des Anteils erneuerbarer Energie in der EU von derzeit $6 \%$ auf $12 \%$ erreicht werden, was wiederum ein wesentlicher Beitrag zur Erfüllung der Kyotoverpflichtungen wäre.

Eine ganz wichtige Voraussetzung zur Erreichung des Gesamtziels ist, daß sich die Mitgliedsländer nationale Ziele für 2005 und 2010 setzen und auch Strategien zu ihrer Erreichung ausarbeiten. Italien hat bereits beispielhaft ein 10000-Photovoltaik-Dächer-Programm verkündet und Großbritannien hat sich vorgenommen, seinen minimalen Anteil von 0,7\% erneuerbarer Energie bis 2010 auf respektable $10 \%$ zu erhöhen. Und dort sind Biomasse und Wasserkraft Mangelware im Vergleich zu Österreich.

Auch Österreich ist aufgerufen, über ein nationales Ziel nachzudenken, zumindest etwa Erhöhung des Anteils erneuerbarer Energie um 6 Prozentpunkte bis 2010. 